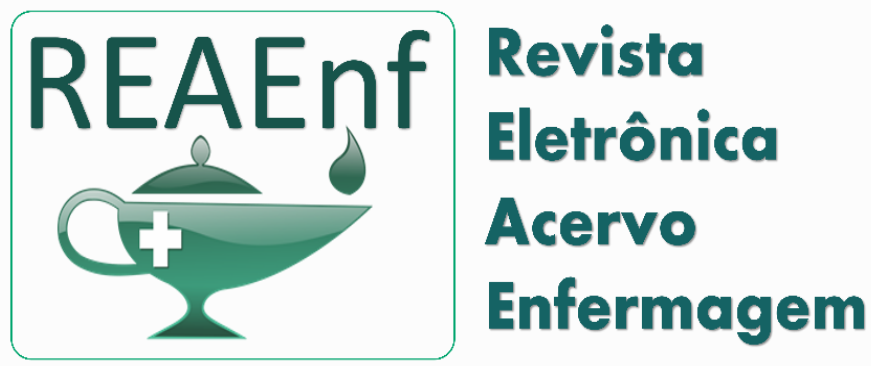

REVISÃO BILIOGRÁFICA

Recebido em: 11/2020

Aceito em: 12/2020

Publicado em: 1/2021

\title{
Adesão dos profissionais de enfermagem as metas de segurança da OMS: uma revisão de literatura
}

\author{
Adherence of nursing professionals to security targets of the WHO: a bibliographic review \\ Adhesión de los profesionales de enfermería a los objetivos de seguridad de la OMS: \\ revisión de la literatura
}

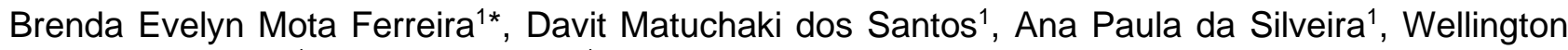
Ferreira de Souza ${ }^{1}$, Francieli Carniel ${ }^{1}$.
\end{abstract}

Resumo: Este artigo teve por objetivo analisar se os protocolos de segurança do paciente são aderidos pela equipe de enfermagem, identificando as lacunas que dificultam essa adesão. Trata-se de uma revisão integrativa de literatura. Para análise dos dados foram selecionados 32 artigos para compor a amostra, destes, 26 mostraram baixa aplicação na prática profissional e apenas 14 apresentaram grau de conformidade satisfatório, classificando a adesão da equipe aos protocolos de segurança como indesejada. Em relação as dificuldades apontadas que levam a baixa adesão da equipe destacam-se sobrecarga de trabalho, deficiência estrutural, resistência da equipe, dimensionamento de recursos e de pessoal inadequado. Evidenciou-se que embora haja o reconhecimento pela equipe de enfermagem à cerca da importância dessa temática, ainda presencia- se o descompasso entre a teoria e a prática, demonstrado pela baixa adesão aos protocolos de segurança, sendo essencial, além da padronização desses protocolos, a elaboração de estratégias como fiscalização e auditoria de processos, objetivando reduzir os riscos e permitindo a melhoria na segurança e a qualidades na assistência em saúde.

Palavras-chave: Qualidade da assistência, Segurança do paciente, Protocolos.

Abstract: This article aimed analyzes whether patient safety protocols are adhered to by the nursing staff, identifying the gaps which complicate that accession. It is an integrative literature review. For data analysis 32 articles were selected to compose the sample, of these 26 demonstrated low applications in professional practice and only 14 showed satisfactory degree of compliance, classifying the team's agreement to safety protocols as undesirable. In relation to the difficulties identified that lead to low group adherence, the work overload, structural deficiency, group resistance and the inadequate dimensioning of resources and personnel are highlighted. It was explicit that even though there is recognition by the nursing team about the importance of this theme, there's still a gap between theory and practice, demonstrated by the inadequate adherence to security protocols, being essential, in addition to the standardization of them, the elaboration of strategies such as oversight and auditing of processes, aiming to reduce risks and allowing the improvement in safety and quality in health care.

Keywords: Quality of health care, Patient safety, Protocols.

Resumen: Este artículo tuvo como objetivo analizar si el equipo de enfermería cumple con los protocolos de seguridad del paciente, identificando las brechas que dificultan está adherencia. Es una revisión integradora de la literatura. Para el análisis de los datos se seleccionaron 32 artículos para componer la muestra, de estos

${ }^{1}$ Centro Universitário São Lucas, Ji-Paraná - RO. *E-mail: brendaevelynmf@gmail.com

REAEnf/EJNC | Vol. 8 | e5967 | DOI: https://doi.org/10.25248/REAenf.e5967.2021 Página 1 de 8 
26 presentaron baja aplicación en la práctica profesional y solo 14 tuvieron un grado de cumplimiento satisfactorio, clasificando como indeseable la adherencia del equipo a los protocolos de seguridad. En relación a las dificultades identificadas que conducen a baja adherencia al equipo, se destaca la sobrecarga de trabajo, deficiencia estructural, resistencias del equipo, dimensionamiento de recursos y personal inadecuado. Se evidenció que si bien existe un reconocimiento por parte del equipo de enfermería sobre la importancia de este tema, aún existe una brecha entre la teoría y la práctica, demostrada por la baja adherencia a los protocolos de seguridad, siendo fundamental, además de la estandarización de estos protocolos, la elaboración de estrategias como la inspección y auditoría de procesos, con el objetivo de reducir riesgos y permitir la mejora de la seguridad y calidad en la atención de la salud.

Palabras clave: Calidad de la assistência, Seguridad del paciente, Protocolos.

\section{INTRODUÇÃO}

A OMS (2004) define segurança do paciente como "a redução do risco de danos desnecessários a um mínimo aceitável associado ao cuidado de saúde", a mesma definição também foi aderida pela Portaria MS/GM no 529/2013 que estabelece o Programa Nacional de Segurança do Paciente (MS, 2013). Baseado no princípio da beneficência de reduzir os riscos de danos previsíveis ao mínimo possível, as questões associadas a segurança do paciente têm sido reconhecidas mundialmente e representa um grave desafio à saúde pública global, pois nesta área, a incidência de danos aos pacientes é específica ao processo de trabalho e na ocorrência desses, podem acarretar sequelas permanente, aumento do tempo de hospitalização e índice de mortalidade (WHO, 2013).

A Resolução da Diretoria Colegiada (RDC) no. 36 de 25 de julho de 2013 estabelece que os serviços de saúde do país devem implantar os Núcleos de Segurança do Paciente. Como cumprimento do regulamento técnico, os NSP dos serviços de saúde do Brasil realizam mensalmente, a notificação de casos de incidentes que acontecem durante à assistência à saúde no Sistema Nacional de Vigilância Sanitária (MS, 2013). Todavia de acordo com Maia S (2018) no Brasil a incidência dos eventos adversos em saúde é pouco investigada, embora as notificações e registros pelo NSP são obrigatórias desde junho de 2014.

Para se alcançar um assistência segura, a OMS criou o projeto Aliança Mundial para a Segurança do Paciente e com participação da Comissão Conjunta Internacional (Joint Commission International (JCI)) estabeleceu as seis metas internacionais de segurança do paciente, sendo elas: Identificar o paciente corretamente; melhorar a eficácia da comunicação; medicação segura; cirurgia segura; reduzir o risco de infecções associadas a cuidados; reduzir o risco de danos ao paciente decorrente de quedas e lesão por pressão (JCl, 2011; BRASIL, 2014).

Atendendo a proposta da OMS a Portaria MS/GM no 529/2013 estabelece a elaboração e implantação de um conjunto de Protocolos de Segurança do Paciente, definidos pela OMS (MS, 2013), dessa forma a Portaria no 2.095 de setembro de 2013 sugere os protocolos de Prevenção de Quedas, Identificação do Paciente e o de Segurança na Prescrição e de Uso e Administração de Medicamentos (MS,2013), e a Portaria no 1.377 de julho de 2013 propõe os Protocolos de Cirurgia Segura, Prática de Higiene das mãos e Ulcera por Pressão (MS, 2013).

Pesquisas que abordam a temática segurança do paciente e envolvem o enfermeiro para melhoria da qualidade da assistência são recentes e necessários, pois ajudam os profissionais a conhecer as causas e os efeitos à saúde do paciente, permitindo a capacitação adequada com o emprego de roteiros e listas de verificação, utilizando os protocolos para nortear a assistência e prevenir erros e eventos adversos nos serviços de saúde (OLIVEIRA AC, et al., 2017).

Apesar da implantação de protocolos, dedicados a melhoria da assistência saúde, o estudo é relevante pois é necessário conhecer e compreender, as estratégias utilizadas em âmbito hospitalar que tornam os protocolos de segurança efetivo. Dessa forma, por abordar uma temática de extrema relevância questionase: Existe adesão por parte da equipe de enfermagem aos protocolos de segurança do paciente no ambiente hospitalar? Constitui-se como objetivo, analisar se os protocolos de segurança do paciente são aderidos de 
forma efetiva pela equipe de enfermagem identificando as lacunas que dificultam essa adesão, através de revisão de literatura.

\section{MÉTODOS}

Essa pesquisa tem como proposta metodológica uma revisão integrativa de literatura, que permite a análise de estudos já publicados, principalmente de livros e artigos científicos, possibilitando a discussão e conclusão a respeito de um determinado tema ou área de estudo.

Para identificação e seleção dos estudos foi realizado um levantamento bibliográfico com publicações das bases de dados: Literatura Latino-Americana de Ciências da Saúde (LILACS); Scientific Eletronic Library Online (SciELO); Base de dados de enfermagem (BDENF); Medical Literature Analysis and Retrieval System Online (MEDLINE); entre os meses de agosto e setembro de 2020, publicados nos últimos 5 anos (20152020). Para a pesquisa desses artigos foram utilizados os seguintes descritores: "Qualidade da Assistência e Segurança do paciente", "Protocolo, Enfermagem e Segurança do Paciente", "Protocolos e Enfermagem", validados nos Descritores em Ciências da Saúde (DeCS) da BVS.

Como critérios de inclusão e exclusão, foram selecionados artigos que retratem a adesão aos protocolos de segurança, em textos completos disponíveis para análise; publicados em menos de 5 anos, nos idiomas português e inglês e artigos encontrados a partir dos descritores em Ciências da Saúde (DeCS).

Ao concluir a busca nos bancos de dados obteve-se um total de 9186 artigos, após a aplicação dos filtros obteve-se a amostra de 1902 artigos, então procedeu-se uma leitura com ênfase no título de todo material selecionado com o objetivo de verificar se os artigos consultados eram de interesse para a pesquisa, obtendose o total de 79 artigos. Após a aplicação dos critérios de exclusão, análise dos resumos, resultados e conclusões, selecionaram-se 32 artigos, sendo 9 da LILACS, 14 SciELO, 9 da BDENF e 7 da MEDLINE, conforme apresentados na Figura 1. Procedeu-se à leitura interpretativa, baseada nas ideias centrais do artigo identificadas através de perguntas norteadoras como: Os Protocolos são aderidos pela equipe de enfermagem? Qual Protocolo obteve maior adesão pelos profissionais? Qual motivo da baixa adesão aos protocolos?

Os dados foram organizados e armazenados em um instrumento desenvolvido pelos autores, separados por título, periódico, autor, ano de publicação, país do estudo e meta abordada, o que permitiu a organização dos estudos em tabelas possibilitando a melhor compreensão das temáticas e das informações mais importantes para essa revisão, como serão apresentadas na Figura 1.

Figura 1 - Fluxograma da seleção dos artigos.

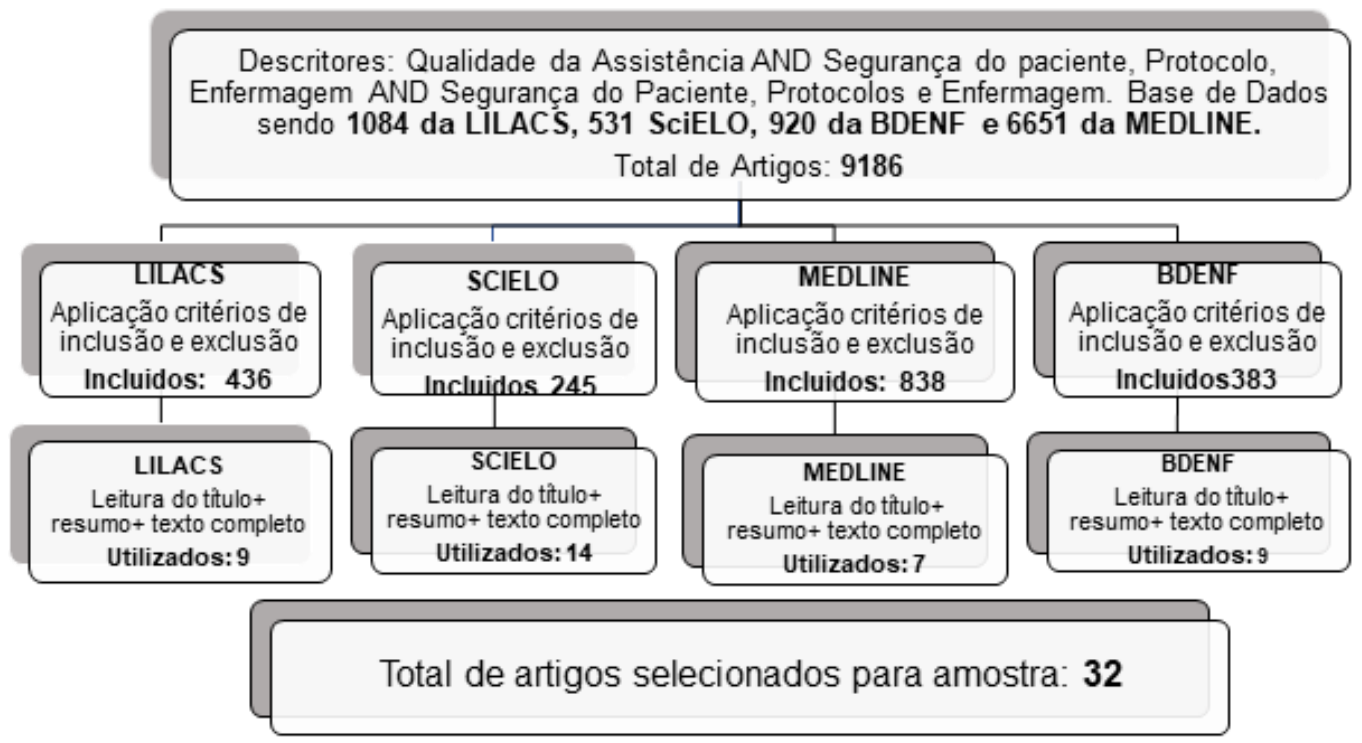

Fonte: Ferreira BEM, et al.,2020. 


\section{RESULTADOS}

Como resultados da busca foram selecionados 32 artigos e utilizados para compor a amostra, dos quais $26(84,38 / \%)$ foram publicados em português e $5(15,62 \%)$ em inglês. Os países onde os estudos foram realizados são: Finlândia 1 (3,12\%), Grécia $1(3,12 \%)$, Holanda 1 (3,12\%), Estados Unidos da América (EUA) $1(3,12 \%)$, Malawi $1(3,12 \%)$ e Brasil $27(84,33 \%)$. Dos artigos que foram escritos no Brasil foi da região Norte $1(3,70 \%)$, Nordeste $8(29,62 \%)$ e da região Sul $7(25,92 \%)$, Sudeste $10(37,03)$ e Centro Oeste $1(3,70 \%)$. Em relação ao ano de publicação dos estudos o ano de 2017 teve maior destaque com 11 (34,37\%) seguido de 2019 com 8 (25\%), 2018 com 6 (18,75\%), 2015 com 2 (6,25\%) e 2020 com 5 (15,62\%) dos artigos publicados.

Quanto ao contexto em que se realizaram os estudos, todos foram em ambiente hospitalar, totalizando 51 hospitais, visto que alguns foram realizados em mais de um hospital destes, $5(9,80 \%)$ foram em hospitais universitários, 2 (3,92\%) na maternidade, 5 (9,80\%) em UTIs, 4 (7,84\%) no Centro Cirúrgico, 2 (3,92\%) em Clínicas Médicas, 1 (1,96\%) em uma Unidade de Pronto Atendimento (UPA) e $32(62,74 \%)$ não foram especificadas as clínicas de realização do estudo, podendo-se presumir que abrangeram todo ambiente hospitalar.

No que se refere as metas abordadas destaca-se com maior percentual de artigos a de Cirurgia Segura, seguido de Prevenção de Lesão por Pressão e Quedas, Segurança na Prescrição Uso e Administração de Medicamentos, Prática de Higiene das Mãos, Comunicação Efetiva, Identificação do Paciente e os artigos que abordaram todas as metas, conforme exposto no Gráfico 2.

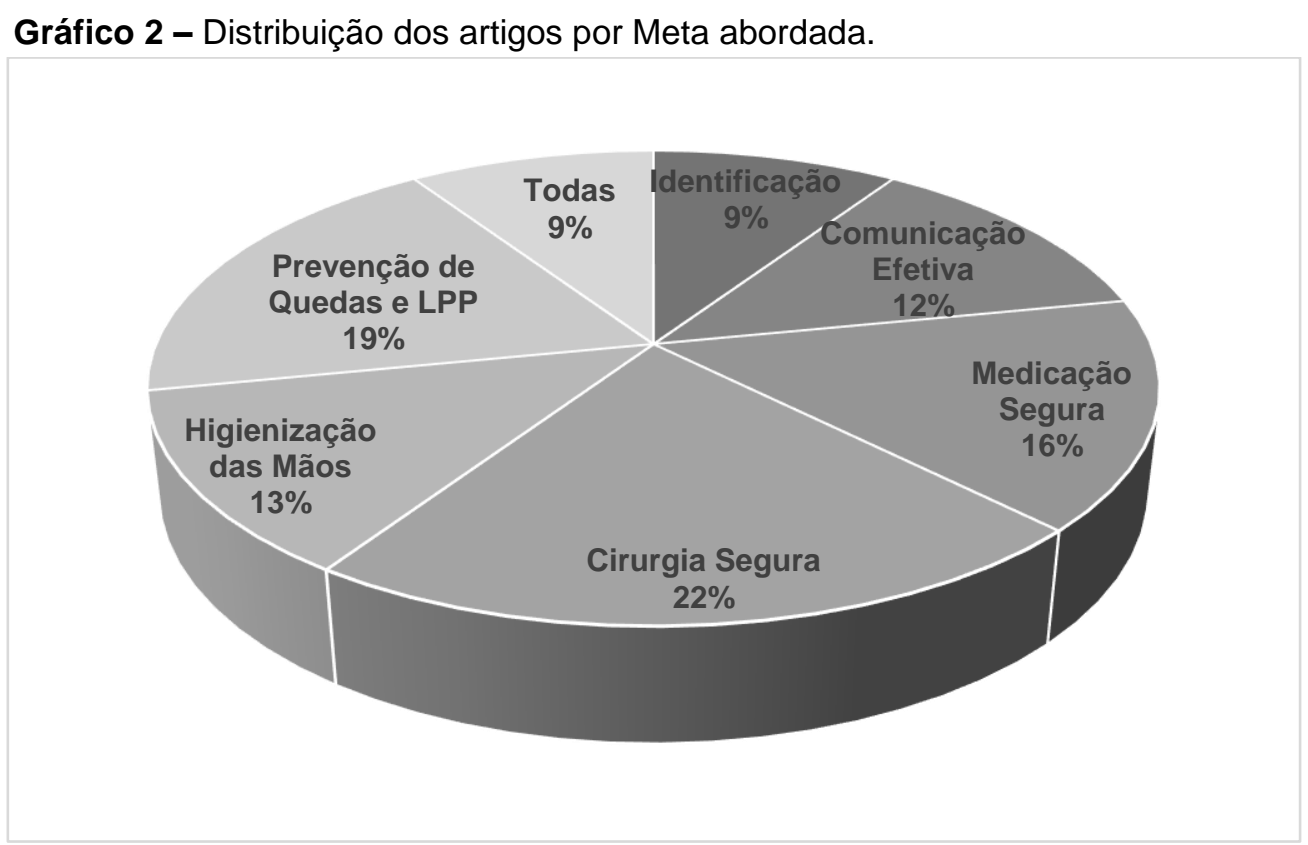

Fonte: Ferreira BEM, et al., 2020.

Ao observar a adesão da equipe aos protocolos elaborados a partir das metas, evidenciou-se que, no geral a adesão da equipe foi classificada como sofrível ou indesejada, considerando que dentre os 32 estudos, foram analisados 40 resultados de adesão aos protocolos, visto que alguns estudos abordaram mais de um protocolo, destes, $26(65 \%)$ mostraram baixa aplicação na prática profissional e apenas 14 (35\%), apresentaram grau de conformidade satisfatório. O protocolo de Prevenção de quedas e Lesão por Pressão teve maior adesão com $66,66 \%$ de conformidade e o de Medicação segura apresentou-se insatisfatório com $90 \%$ de inconsistência.

Os artigos foram categorizados quanto a abordagem metodológica e o objetivo adotado, sendo: 11 $(34,37 \%)$ estudos quantitativos, $6(18,75 \%)$ qualitativos e $15(46,8 \%)$ estudos não tiveram o tipo de pesquisa 
bem esclarecido. Em relação ao delineamento dos estudos foram assim distribuídos: 17 (53,12\%) descritivo, $1(3,12 \%)$ explicativo, $4(12,48 \%)$ descritivo exploratório, 1 (3,12\%) descritivo explicativo e $9(28,13 \%)$ o objetivo não foi bem explícito.

A Quadro 1 mostra algumas dificuldades apontadas pela equipe de enfermagem como razão para as baixas taxas de adesão as recomendações, expondo as fragilidades no sistema de saúde que comprometem uma assistência de qualidade, refletindo o marcante distanciamento entre o cuidado real e o cuidado ideal.

Quadro 1 - Dificuldades apontadas para adesão aos protocolos de segurança de acordo com cada meta.

\begin{tabular}{|c|c|}
\hline Meta & Dificuldades \\
\hline Identificação correta & $\begin{array}{l}\text { A sobrecarga de trabalho, dimensionamento inadequado dos } \\
\text { profissionais de enfermagem, falta de materiais e a falta de comunicação } \\
\text { (SILVA RS, et al., 2019; SIMAN AG, et al., 2020; ZAMPOLLO N, et } \\
\text { al.,2018); }\end{array}$ \\
\hline Comunicação efetiva & $\begin{array}{l}\text { Falta de padronização e familiarização dos profissionais com as rotinas } \\
\text { (PETRY L, et al.,2019; SIMAN AG, et al., 2020); }\end{array}$ \\
\hline Medicação segura & $\begin{array}{l}\text { Carga horária, exaustão, inexperiência, déficits de conhecimento, } \\
\text { comunicação pobre e uma cultura do silêncio, conflito entre horários de } \\
\text { administração de medicamentos e rotinas de enfermaria, curto tempo } \\
\text { para executar as tarefas, o esquecimento, a falta de observação de } \\
\text { atitudes para assistência segura, a escassez de recursos humanos } \\
\text { (MULA CT, et al., 2020; SCHUTIJSER B, et al., 2017; ZAMPOLLO N, et } \\
\text { al., 2018; OLIVEIRA JKA, et al., 2018); }\end{array}$ \\
\hline Cirurgia segura & $\begin{array}{l}\text { Falta de participação da equipe, emprego de itens de difícil compreensão, } \\
\text { ausência de explicação sobre a lista de verificação e falta de tempo para } \\
\text { o preenchimento, dificuldade na comunicação, sobrecarga de trabalho, } \\
\text { falta de comunicação da equipe, falta de capacitação e resistência da } \\
\text { equipe cirúrgica (SANTOS EA, et AL., 2020; SILVA FAA, et al., 2017; } \\
\text { GARCIA TF, et al., 2018; GUTIERRES LS, et al., 2019); }\end{array}$ \\
\hline Higiene das mãos & $\begin{array}{l}\text { Esgotamento da equipe, alta carga de trabalho, falta de pessoal, falta de } \\
\text { tempo, dificuldade de acesso às pias e a falta de desinfetantes para as } \\
\text { mãos devidamente localizados no ponto de atendimento e estrutura física } \\
\text { inadequada (MANOMENIDIS GMS, et al., 2019; SANDULE-RIOS N, et } \\
\text { al., 2017) }\end{array}$ \\
\hline $\begin{array}{l}\text { Prevenção de quedas e Lesão } \\
\text { por pressão }\end{array}$ & $\begin{array}{l}\text { Dimensionamento inadequado, desgaste, sobrecarga e estresse, falta de } \\
\text { educação permanente e comunicação efetiva (HOLANDA OQ, et al, } \\
\text { 2018; SOUZA MC, et al., 2020) }\end{array}$ \\
\hline
\end{tabular}

Fonte: Ferreira BEM, et al., 2020.

\section{DISCUSSÃO}

Diante do intuito de investigar a adesão da equipe de enfermagem, foram estabelecidas duas categorias que permitem melhor compreensão da temática abordada: 1) Adesão aos protocolos 2) Motivos para a baixa adesão.

\section{Os Protocolos são aderidos pela equipe de enfermagem?}

A Enfermagem é regulamentada pela Lei 7.498/1986, que dispõe em parágrafo único que sua equipe é composta pelo Enfermeiro, pelo Técnico de Enfermagem, pelo Auxiliar de Enfermagem e pela Parteira, respeitando os níveis de habilidades (BRASIL, 1986).

Os Profissionais de enfermagem são as principais fontes das ações assistenciais, e prestam cuidados direto ao paciente, o que aponta a necessidade de adoção e implementação de estratégias de segurança que 
visam reduzir a possibilidade de incidentes que atingem o paciente, tais como queda do paciente, lesões por pressão, erros de medicação, queimaduras durante procedimentos, extubação, erros em hemotransfusões, infecção, hemorragias por desconexão de drenos e cateteres (LIMA BB e BRUM AKR, 2016).

A primeira meta de segurança do paciente da OMS trata-se de identificar corretamente o paciente pois 0 processo de identificação incorreta do paciente leva o aumento do índice de eventos adversos (ANVISA, 2017). Estudos que abordam os protocolos de identificação correta ressaltam que a identificação dos pacientes não foi totalmente utilizada e a pulseira não é checada antes de procedimentos (SILVA RS, et al., 2019). Em contrapartida outro estudo demostrou alta adesão da equipe de enfermagem ao protocolo de identificação, porém algumas inconformidades foram citadas, principalmente a ilegibilidade da pulseira (ZAMPOLLO N, et al., 2018).

A segunda meta refere-se em melhorar a comunicação entre os profissionais de saúde pois a comunicação efetiva principalmente na área hospitalar é um processo complexo e dinâmico, por se tratar de um ambiente com um grande fluxo de informações e com equipe multiprofissional com grande demanda de atividades que necessitam de constante troca de informações. Petry L (2019), estabelece que o processo de comunicação ocorre de maneira frágil e apresenta diversas lacunas decorrentes da inexistência de um protocolo e do pouco reconhecimento acerca da importância por parte dos profissionais envolvidos.

Melhorar a segurança na prescrição, no uso e na administração de medicamentos, corresponde a terceira meta de segurança visto que os erros na administração de medicamentos e os eventos adversos estão entre as falhas mais frequentes relacionadas aos cuidados em saúde, sendo importante destacar que são situações que podem ser evitadas (ANVISA, 2013).

Estudos apontam que a maioria dos procedimentos são executados por técnicos de enfermagem e que entre os nove certos da administração de medicamentos, a equipe mencionou erros aos itens: medicamento, dose, horário e orientação certa e demonstram maior adesão aos itens forma e via certa (LLAPARODRIGUES EO, et al., 2018).

Em conformidade com isso, um estudo realizado em um hospital de Malawi demonstrou que a equipe de enfermagem enfrenta desafios com o cumprimento de administrar na hora certa e dar a dose certa, comprometendo a eficácia dos antibióticos, os desafios foram relacionados aos horários de rotina de administração de medicamentos, horário de visita e quando o paciente está dormindo, demonstrando pouco conhecimento de como os antibióticos funcionam; e falta de pensamento crítico quanto ao horário de visitas e que e o sono tem prioridade sobre a administração de antibióticos (MULA CT, et al.,2019).

A quarta meta visa garantir que as cirurgias sejam feitas no local correto com procedimento correto e no paciente correto com objetivo de prevenir erros tais como: cirurgias em locais errados, complicações anestésicas, intercorrências durante o procedimento e complicações no pós-operatório (BRASIL, 2009). Os checklists elaborados no protocolo de cirurgia segura é a principal estratégia para prevenção desses eventos, embora conforme um estudo realizado em um hospital universitário de Minas Gerais, na prática profissional a implementação desse checklist cirúrgico vem apresentando falhas na sua aplicação como, falta de identificação dos pacientes e confirmação do procedimento realizado, não apresentação da equipe cirúrgica e monitorização da temperatura, e quando as etapas são realizadas, não atendem ao que de fato está proposto para cada uma delas (OLIVEIRA AC, et al., 2017).

No que se refere a higienizar as mãos para evitar infecção a OMS elabora a quinta meta com objetivo de reduzir infecções e promover segurança de pacientes, profissionais e usuários. Em estudo realizado em Sergipe, evidenciando que mesmo sendo muito divulgada, a adesão à prática de higienização das mãos ainda não se encontra dentro dos padrões esperados, pois os profissionais de enfermagem, apesar de reconhecer a importância dessa medida, não à coloca em prática, tornando-se um grande desafio para o controle das infecções relacionadas à assistência à saúde (OLIVEIRA JKA, et al., 2018)

A sexta meta propõe reduzir o risco de quedas e lesões por pressão (LPP), que representam um desafio para a assistência à saúde, pois além de acarretarem uma série de danos aos pacientes, também são responsáveis por aumento nos custos financeiros dos serviços de saúde. No entanto em muitos casos esses

REAEnf/EJNC | Vol. 8 | e5967 | DOI: https://doi.org/10.25248/REAenf.e5967.2021 Página 6 de 8 
incidentes podem ser evitáveis, se adotadas medidas de prevenção tais como, avaliação por meio de escalas, uso de instrumentos e protocolos institucionais (ANVISA, 2017)

Embora um estudo realizado em 3 hospitais públicos de Campo Grande em Mato Grosso do Sul que teve como alvo de estudo toda a equipe de enfermagem, mostrou baixo conhecimento por parte dos profissionais acerca da existência de protocolos institucionais para prevenção do desenvolvimento de LPP (SOUZA MC, et al., 2020).

Outro evento adverso que pode ser completamente evitável através da prevenção, são os incidentes por quedas, que são uma das causas mais comum de danos, provocando aumento de lesões teciduais, fraturas e até mesmo o óbito, principalmente em idosos hospitalizados (COSTA SGRF, et al., 2011).

De acordo com Alves VC (2017), a priorização de cuidados relacionados às "práticas diretas para prevenção", como: identificar os pacientes com alto risco para quedas e os alocar próximo ao posto de enfermagem; movimentar os pacientes de forma segura; e agendar cuidados de higiene pessoal e horários regulares para levar o paciente ao banheiro. Em contrapartida com isso outro estudo realizado em uma Unidade de Pronto Atendimento em Curitiba no Paraná, verificou-se não haver avaliação rotineira do risco para o seu desenvolvimento (PAIXÃO DPSS, et al., 2017).

\section{Qual motivo da baixa adesão aos protocolos?}

Em geral a maior parte dos estudos apontou como dificuldades para adesão dos protocolos a sobrecarga de trabalho. Corroborando com esse ponto de vista, Silva FAA (2017) considera que a quantidade de atividades realizadas pela equipe de enfermagem é um fator que dificulta a realização de ações direcionadas a segurança do paciente, pois frequentemente na pretensão de executar todas as atividades diárias, algumas práticas seguras acabam sendo excluídas da rotina, tais como, uso de luvas, desinfecção das mãos frequentemente, mudança de decúbito dos pacientes, desinfeç̧ão das ampolas, identificação dos medicamentos, elevação das grades dos leitos, dentre outras.

A falta de tempo apontada como dificuldade para adesão ao protocolo de cirurgia segura é oposta as características apresentadas sobre as listas de verificação e check lists que são ferramentas que permitem um rápido preenchimento e interpretação da situação. De acordo com Santos EA (2020) essa barreira se fortalece pela falta de participação de toda a equipe gerando sobrecarga de responsabilidades e atribuições a um único membro da equipe. Outra dificuldade apontada trata-se de particularidades do próprio pessoal de enfermagem, como não envolvimento e resistência da equipe às estratégias para segurança do paciente, eventualmente por terem criado uma cultura de serviço e desenvolvido hábitos durante todo tempo de emprego, que tornam difíceis de serem trabalhados (SANTOS EA, et al., 2020).

A falta de estrutura adequada, como a dificuldade de acesso aos recursos de higiene das mãos, ausência de materiais, equipamentos velhos e instalações antigas, também foram citadas nos estudos e são características do sistema de saúde que influenciam as ações da equipe de enfermagem, pois, de acordo com Báo AC (2019), a assistência de qualidade por parte dos profissionais de saúde dependem extremamente de equipamentos, instalações, materiais e recursos humanos, de modo que a carência comprometem o trabalho das equipes e os seus resultados. Dessa forma o empenho para sanar essas dificuldades, deve ser proveniente de todas as direções, promovendo estrutura física, humana e organizacional que garanta uma assistência de qualidade dos profissionais. A pesquisa apresentou limitações relacionadas a falta de estudos que abordam a adesão da equipe de enfermagem aos protocolos de segurança, dificultando obter uma amostra mais significativa. Outra limitação diz respeito aos locais onde foram realizados os estudos, pois a maioria foram em hospitais públicos, não permitindo fazer uma comparação da adesão e dificuldades apontadas pelos profissionais na rede pública e privada.

\section{CONSIDERAÇÕES FINAIS}

Evidenciou-se na presente pesquisa, que embora os serviços venham adotando medidas de maneira a assistir a segurança do paciente e se tratando de uma temática de extrema relevância que tem conquistado dimensões significativas, ainda se percebe que apesar da elaboração de todos os protocolos, há lacunas no 
que se refere a implementação efetiva e monitoramento destes, por parte da equipe de enfermagem e os demais profissionais de saúde, gestores e pelo próprio usuário. Portanto se faz necessário a educação continuada para melhor envolvimento da equipe, bem como a adoção de metodologias para avaliar a qualidade do serviço, com estratégias como fiscalização, auditoria de processos além de instituir melhorias quanto de estrutura física e disponibilidade de materiais adequados, tais medidas poderão contribuir para redução de riscos e permitir a segurança e a qualidades na assistência em saúde.

\section{REFERÊNCIAS}

1. ALVES VC, et al. Ações do protocolo prevenção de quedas: mapeamento com a classificação de intervenções de enfermagem. Revista Latino-Americana de Enfermagem, 2017; 25: 1-12.

2. ANVISA. Agência Nacional De Vigilância Sanitária. Assistência Segura: Uma Reflexão Teórica Aplicada à Prática. Brasília, 2017.

3. ANVISA. Agência Nacional De Vigilância Sanitária. Assistência Segura: Uma Reflexão Teórica Aplicada à Prática. Brasília, 2017.

4. ANVISA. Agência Nacional De Vigilância Sanitária. Relatório de autoavaliação nacional das práticas de segurança do paciente em serviços de saúde - 2019. Brasília, 2020.

5. BRASIL, Lei $N^{\circ} 7.498$, de 25 de Junho de 1986. Dispõe sobre a regulamentação do exercício da enfermagem, e dá outras providências. Brasília, 1986.

6. BRASIL, MS. Cirurgias seguras salvam vidas manual. ed.1, Brasília, 2009.

7. BRASIL, Portaria $N^{\circ} 1.377$, de $9^{\circ}$ de Julho de 2013. Aprova os Protocolos de Segurança do Paciente. Brasília, 2013

8. BRASIL, Portaria № 2.095, de 24 de Setembro de 2013. Aprova os Protocolos Básicos de Segurança do Paciente. Brasília, 2013.

9. BRASIL, Portaria № 529, de 1ํ de abril de 2013. Institui o Programa Nacional de Segurança do Paciente (PNSP). Brasília, 2013.

10. BRASIL, Portaria № 529, de 1ํ de abril de 2013. Institui o Programa Nacional de Segurança do Paciente (PNSP). Brasília, 2013.

11. BRASIL, RDC № 36 , de $26^{\circ}$ de Julho de 2013. Institui ações para a segurança do paciente em serviços de saúde e dá outras providências. Brasília, 2013.

12. BRASIL, MS. Protocolo De Segurança Na Prescrição, Uso E Administração De Medicamentos. Brasília, 2013.

13. BRASIL, MS. Documento de referência para o Programa Nacional de Segurança do Paciente. v.40, 1.ed. Brasília, 2014.

14. COSTA SGRF, et al. Caracterização das quedas do leito sofridas por pacientes internados em um hospital universitário. Revista Gaúcha Enfermagem, 2011; 32: 676-681.

15. GUTIERRES LS, et al. Adesão aos objetivos do Programa Cirurgias Seguras Salvam Vidas: perspectiva de enfermeiros. Revista Latino-Americana de Enfermagem, 2019; 27: 1-10.

16. JCl, Joint Commission International. Padrões de acreditação para Hospitais. Consórcio Brasileiro de Acreditação de Sistemas e Serviços de Saúde - Rio de Janeiro: 2011.

17. KORHONEN A, et al. Aderência às diretrizes de higiene das mãos - importância de medir a fidelidade. JCN Clinical Nursing, 2015; 24: 3197-3205.

18. LIMA BB, BRUM AKR. Prevenção de queda em paciente hospitalizado e a segurança do paciente: revisão integrativa. Revista enfermagem atual in derme,2016; 78: 36-41.

19. LLAPA-RODRIGUEZ EO, et al. Assistência segura ao paciente no preparo e administração de medicamentos. Revista Gaúcha Enfermagem, 2019; 38: 1-9.

20. MAIA S, et al. Notificações de eventos adversos relacionados com a assistência à saúde que levaram a óbitos no Brasil, 2014-2016. Epidemiol. serv. saúde, 2018; 23: 1-10.

21. MULA CT, et al. O exame da adesão das enfermeiras aos 'cinco direitos' da administração de antibióticos e os fatores que influenciam suas práticas: um estudo de caso de métodos mistos em um hospital terciário, Malawi. Malawi Medical Journal, 2019; 31: 1-7.

22. OLIVEIRA AC, ABREU AR. Implementação do checklist de cirurgia segura em um hospital universitário. Enfermagem em Foco, 2017; 8: 14-18.

23. OLIVEIRA JKA, et al. Segurança do paciente na assistência de enfermagem durante a administração de medicamentos. Revista Latino-Americana de Enfermagem, 2018; 26: 1-9.

24. PAIXÃO DPSS, et al. Adesão aos protocolos de segurança do paciente em unidades de pronto atendimento. Revista Brasileira de Enfermagem, 2017; 73: 622-629.

25. PETRY L, DINIZ MBC. Comunicação entre a equipe e transferência de cuidados de pacientes críticos. Revista Rene. 2019; 21: 1-8.

26. SILVA RSS, et al. Uso de pulseiras de identificação: implicações para a segurança do recém-nascido na maternidade. Escola Anna Nery, 2019; 23: 1-6.

27. SOUZA MC, et al. Cultura organizacional: prevenção, tratamento e gerenciamento de risco da lesão por pressão. Revista Brasileira de Enfermagem, 2020; 73: 1-7.

28. WHO, World Health Organization. The Second Global Patient Safety Challenge: Safe Surgery Saves Lives. World Alliance for Patient Safety: Genebra, 2013.

29. ZAMPOLLO N, et al. Adesão ao protocolo de identificação do paciente e medicação segura. Revista de enfermagem UFPE on-line, 2018; 1-8. 